\title{
Editor's Note for the Proceedings of the Artificial Intelligence for Science, Industry and Society - AISIS2019
}

\author{
Gergely Gábor Barnaföld \\ Wigner Research Centre for Physics of the Hungarian Academy of Sciences \\ E-mail: Barnafoldi.Gergelyawigner.hu \\ Lukas Nellen \\ Instituto de Ciencias Nucleares \\ Univesidad Naciona Autónoma de México \\ E-mail: lukas@nucleares.unam.mx
}

\section{Guy Paić}

Instituto de Ciencias Nucleares

Univesidad Naciona Autónoma de México

E-mail: Guy.Paic@cern.ch 
"By far, the greatest danger of Artificial Intelligence is that people conclude too early that they understand it." —Eliezer Yudkowsky

An exceptional symposium took place at the Universidad Nacional Autonoma de Mexico (UNAM) on the impact of artificial intelligence on science, industry and society with 185 participants of various fields. The event was jointly organized by the UNAM, CERN Openlab. We aimed to discuss the prospects, possible effects, and natural reactions of the mankind of this new and emerging field. The symposium had an exciting program including about 50 presentation, a policy session moderated by the OECD with two panels of policy makers, industry representatives, and a student tutorial on neural networks.

\section{Aim and scope}

What will we be able to do with computers that learn, or computer that work at the very basic quantum foundation of our world? How will these new emerging technologies impact our life in the global village?

The exponential increase in computing power we have witnessed during the last decades has been accompanied by an even more explosive increase of the data available, to the point that making sense of this "ocean of data" could be the major driver of innovation in the years to come. Artificial Intelligence (AI) is emerging as one of the most promising avenues to analyse and extract useful information from a large amount of data.

$\mathrm{AI}$ is not a new concept and it is present in many aspects of our life, from assisting radiologists too recognise cancer in medical imagery to helping particle physicists analyse complex events.

However, the recent developments Deep Learning, coupled with High Performance Computing (HPC) open completely new and futuristic perspectives. Will robot-scientists be able to master the entire chain from generating scientific hypotheses by mining academic literature, performing the appropriate simulations and experiments all the way up to publishing scientific results? Initial steps in this direction have already been taken by King et al. in genomics and tropical disease research. Can it go even further, filing patents on the path to commercialisation?

The industry is since long harnessing AI for applications including robotized production lines, mobile device apps, autonomous vehicles, and many more. Will the AI of the future go even further and anticipate customer needs, design new products and market them?

It is important to distinguish between weak AI and strong AI. Weak AI reproduces what it has learnt in a relatively restricted scope, can perform well within that scope but still stays easily controllable by humans who program its scope and control the boundaries of its action. Strong AI, on the other hand, acts more like a brain, constantly adapting its scope to the external stimuli encountered. It can make connections between apparently unrelated concepts, and go beyond human cognitive biases. Strong AI can become much more powerful at problem solving, but could potentially escape human control and become hostile to humans.

Some specialists argue that resolving societal challenges such as defeating illness or even death, solving climate and environmental issues, will need the power of strong AI. This will imply risk assessment and different scenarios to keep humans in control while leveraging the new opportunities. 
These questions raise ethical and societal concerns, which need to be addressed in order to ensure transparency, accountability, governance by humans, security, safety,as well the right balance between privacy and transparency ('as open as possible, as closed as necessary'). The overall context of digitisation, open Science and open Innovation requires new thinking in terms of policies.This accelerating IT revolution is increasingly at the cross-road of all walks of science, technology, society, philosophy and politics.

In order to harness successfully these new technologies, it is necessary to create spaces for multidisciplinary exchange between basic research, advanced IT providers, potential users in different fields and political deciders.

It is in this context that we organized the Symposium addressing in an interdisciplinary approach the present state and future developments on the fundamental issues, the industrial applications and the societal aspects:

The editors of this PoS volume would like to express their thanks for the support of our sponsors: IBM SinergiaSys, HUAWEI Technologies - MR Solutions, ATOS, Nvidia, Veeam LUFAC, Tline, Dell Technologies. Their participation and sponsorship made the Symposium a successful event.

\section{Committees}

\subsection{Scientific Committee}

- Alcubierre Miguel, ICN-UNAM

- Barnaföldi Gergely Gábor, Wigner Research Centre for Physics

- Benítez Héctor, IIMAS-UNAM

- Bracho Felipe, DGTIC-UNAM

- Carminati Federico, CERN, co-chair

- Escalante Ramírez Boris, CViCom-UNAM

- Faggella Dan, Emerj

- Girone Maria, CERN

- Guellec Dominique, OECD

- Haake Rudiger, Yale

- Ivezić Zeljko, University of Washington

- Lee William, IA-UNAM

- Loncarić Sven, University of Zagreb

- Mayo Rafael, CIEMAT

- Antelis Javier, Tecnológico de Monterrey

- Nellen Lukas, ICN-UNAM (co-chair)

- Paic Alan, OECD

- Paić Guy, ICN-UNAM (co-chair)

- Pinto David, BUAP

- Pisanty Alejandro, UNAM

- Schramm Steven, University of Geneva (co-chair)

- Stoye Markus, Imperial College London \& Data Science Institute 
- Valenzuela Octavio, IA-UNAM

- Vallecorsa Sofía, CERN

- Wang Xin-Nian, LBNL

\subsection{Local Organizing Committee}

- Cárdenas Héctor, IQ-UNAM

- Díaz Luciano, ICN-UNAM

- Mora Elsa, ICN-UNAM

- Murrieta Eduardo, ICN-UNAM

- Nellen Lukas, ICN-UNAM

- Paić Guy, ICN-UNAM

- Romo Fabián, DGTIC-UNAM

\section{Sponsors}

\subsection{Gold sponsors}

ATOS, IBM - SinergiaSys, Huawei Technologies - MR Solutions
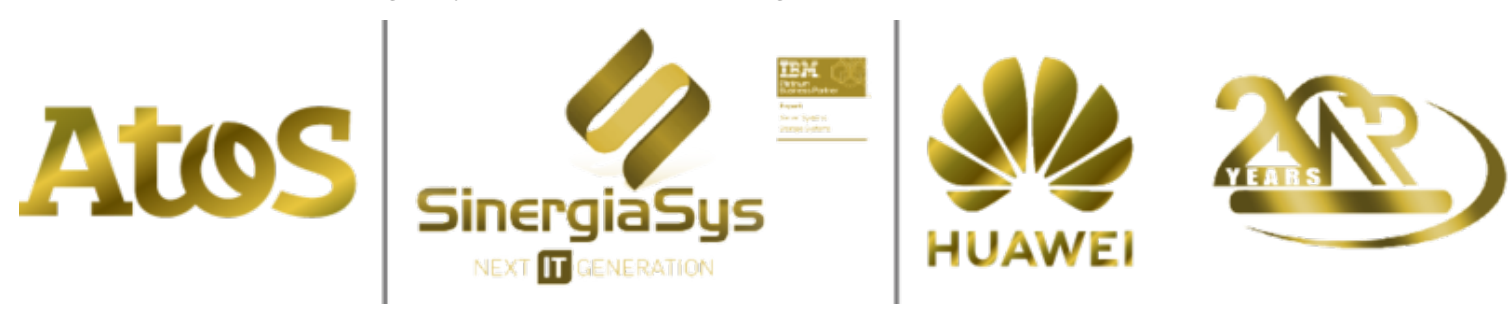

\subsection{Silver sponsors}

NVidia, VEEAM, LUFAC
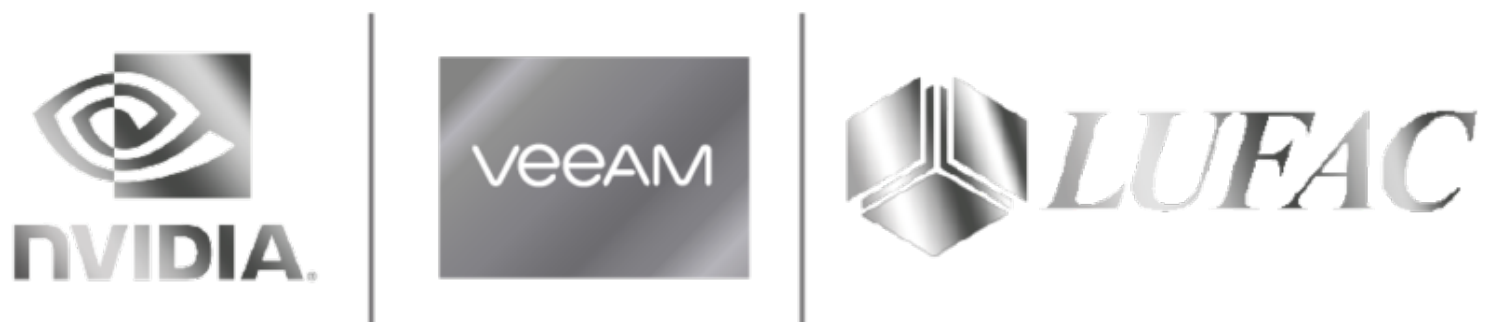

\subsection{Sponsors}

TLine - DELL
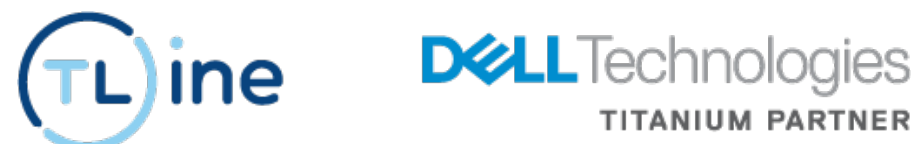

\subsection{Institutional support}

Cern Openlab, CTIC-UNAM, ICN-UNAM, IIMAS-UNAM, IA-UNAM, CVICOM-UNAM, DGTIC-UNAM, FC-UNAM 